tre. En évoquant la nécessaire décentralisation des pouvoirs, l'auteur écrit que la ruralité n'a que des amis qui lui veulent du bien. Ouf! On sait parfois qu'avec certains amis on peut se priver d'ennemis. Passons. En se positionnant parmi les amis de la ruralité, le lecteur amoureux de celle-ci et de la France profonde ne pourra que se délecter à la lecture de cet ouvrage passionnant.

\section{Bernard Jouve, sous la direction de, Les politiques de déplacements urbains en Europe : l'innovation en question dans cinq villes européennes, Paris, L'Harmattan, 2003, 190 p.}

En quoi le transport urbain en Europe peut-il nous concerner? Pas évident à première vue, mais quand on prend en considération la situation, pas toujours facile, qui prévaut à Montréal et dans la plupart de nos villes moyennes, l'expérience vécue sous d'autres cieux ne peut qu'être d'une certaine utilité, pour ne pas dire d'une utilité certaine. On n'a qu'à imaginer ce cycliste empruntant une des nombreuses pistes, dont Trois-Rivières s'enorgueillit, et qui regarde de façon sceptique passer près de lui un gigantesque autobus de la société de transport en commun complètement vide. Est-ce que ça a du sens, se demandet-il en s'interrogeant sur ce qui se fait ailleurs? Sans en être vraiment conscient, Bernard Jouve, professeur au département de géographie de l'UQAM et titulaire de la Chaire de recherche du Canada en étude des dynamiques territoriales, en prenant la responsabilité de cet ouvrage, avait pour ambition de fournir des réponses à tout cycliste urbain en quête de sens. Pour ce faire, on offre au lecteur l'exemple de Genève, Lyon, Munich, Naples et Suttgart avec, à chaque fois, la contribution d'un spécialiste familier avec chacun de ces exemples.

En signalant que les pouvoirs publics ont surtout investi massivement dans les réseaux lourds de transport collectif (métro, tramway, trains régionaux), Jouve se demande si, en évoquant les politiques de déplacements urbains, en lieu et place de politiques de transports urbains, on sous-entend que l'on pense agir différemment à la fois sur le cadre et sur le contenu des politiques publiques. Est-ce le cas dans les différentes villes étudiées ? C'est la question centrale à laquelle l'ouvrage cherche à répondre. En fait, le lecteur se voit ici offrir le fondement des politiques en matière de déplacements urbains observées dans les cinq villes retenues comme autant d'études de cas.

Un élément qui retient l'attention avec tout ce qui s'écrit et se dit sous d'autres cieux en se rapportant aux fameux budgets participatifs de Porto Alegre, c'est l'importance qu'accorde Jouve à la démocratie participative. Il signale, en effet, que dans la majorité des villes ici étudiées, les politiques mises en place furent accompagnées d'une réflexion approfondie sur la démocratie locale et le rôle qui revient à cette chère société civile, pour parler comme Gramsci ou ses disciples québécois. Or, quand on évoque la participation populaire, on le devine bien, les considérations environnementales ont de meilleures chances d'être prises en compte. Il en est donc question ici et là à travers l'ouvrage. Et c'est ici que l'image du cycliste de Trois-Rivières ou d'ailleurs trouve sa pertinence, comme en témoigne l'exemple de Munich.

Cette ville, qui s'est vu attribuer le prix européen de la mobilité en 2000, s'est distinguée entre autres choses par l'opération "Appelez un vélo» ou «Call-abike ». Il s'agissait, à la faveur d'une promotion très bien conduite, de louer 2000 vélos de couleurs voyantes (ce qui change des sinistres vélos noirs d'Amsterdam) comme moyen de transport public individuel. L'histoire ne dit pas si le casque était fourni. Hélas, malgré un intérêt très rapidement manifesté par les citoyens (ou à cause de cela), l'opération s'est avérée à la fois trop complexe à gérer et non rentable. Car il est vite apparu que, pour joindre les deux bouts, il aurait fallu recourir à la contribution des villes limitrophes (tiens donc !), à défaut de quoi les Munichois se seraient vus forcés de payer pour des services dont bénéficieraient en grande partie des résidants desdites villes (retiens donc !). Doit-on y voir un argument en faveur des fusions municipales ? L'auteur, ignorant de la réalité québécoise, n'a pu faire un rapprochement avec une situation qui nous est bien familière. En fait, l'auteur termine sa contribution en signalant que, malgré la très forte mobilisation qu'elle a générée, la nouvelle politique des déplacements urbains à Munich souffre de l'absence d'un leadership fort de nature à stabiliser et à pérenniser le nouveau mode de régulation politique. Encore une fois, le lecteur québécois peut faire les rapprochements qu'il juge pertinents. L'ouvrage lui fournit d'ailleurs beaucoup d'occasions de le faire. 
Mais, on n'y échappe pas : en Europe comme chez nous, l'auto règne en maître et ce, de plus en plus avec les véhicules dit utilitaires (pour aller faire ses courses au centre d'achats). C'est pourquoi Bernard Jouve écrit qu'il est irréaliste de penser pouvoir modifier les préférences individuelles tant l'aspiration à la propriété privée individuelle en banlieue (avec tondeuse à gazon en prime), associée à la voiture particulière, constitue une tendance lourde des sociétés « modernes ». Les guillemets sont de l'auteur. Évidemment, chacun se reconnaîtra dans cette modernité qui, en fait, caractérise la... post-modernité.

\section{André Joyal}

Université du Québec à Trois-Rivières

\section{Marc Ferro (dir.) Le livre noir du colonialisme, $X V I^{e}-X X I^{e}$ siècle : de l'extermination à la repentance Paris, Robert Laffont, 2003, 843 p.}

Voici un ouvrage réalisé par 21 collaborateurs, spécialistes d'histoire et d'anthropologie culturelle rattachés pour la plupart à l'EHESS, à l'Université de Paris et au CNRS. Celui-ci comprend cinq parties, identifiées clairement dans la table des matières: 1) l'extermination; 2) la traite et l'esclavage; 3) dominations et résistances; 4) le sort des femmes; 5) représentations et discours, ainsi qu'un épilogue : "Qui demande des réparations et pour quels crimes ?».

Les phénomènes de domination, surtout par l'Europe, et de résistance, de la part des populations locales, occupent la majeure partie de l'ouvrage. La colonisation menée par deux autres États est aussi analysée : celle de la Russie dans le Caucase et celle du Japon en Asie du Sud-Est. Or, globalement, que veulent démontrer les auteurs? Quatre points principalement.

\section{Enrichissement de l'Europe}

C'est à compter du XVI ${ }^{\mathrm{e}}$ siècle que l'Europe voulut atteindre directement les régions productrices de denrées (épices, sucre, café, thé) et de matières premières (métaux précieux, soie, coton, tabac, indigo, caoutchouc). L'obtention de ces produits, pensait-on, ferait circuler des capitaux à l'intérieur des États et permet- trait à diverses catégories de personnes d'en tirer des profits. En résulterait à coup sûr un enrichissement des États. Dans cette perspective, la formation d'Empires coloniaux allait constituer un moyen par excellence pour en faciliter la réalisation.

Voilà pourquoi, dès le début des temps modernes, entre les années 1450 et 1600, cinq États européens, soit le Portugal, l'Espagne, la Hollande, l'Angleterre et la France, se lancèrent successivement dans la course aux explorations maritimes, puis constituèrent chacun un immense Empire colonial, tantôt dans le Nouveau Monde, tantôt en Asie, parfois dans ces deux régions comme ce fut le cas pour l'Espagne, l'Angleterre et la France. S'y ajoutèrent au même moment des comptoirs de commerce en Afrique, en Chine et au Japon. Plus tard, aux $\mathrm{XIX}^{\mathrm{e}}$ et $\mathrm{XX}^{\mathrm{e}}$ siècles, la plupart de ces États, auxquels s'ajouta la Belgique, exploitèrent en outre systématiquement le continent africain.

Ainsi, selon les auteurs de l'ouvrage, plus de la moitié de la planète allait être sous le joug de l'Europe pendant cinq siècles. D'où la deuxième question qu'ils posent : comment s'y est-on pris pour exploiter et dominer ces immenses régions déjà habitées depuis des millénaires par des populations autochtones?

\section{Modes d'exploitation des Empires coloniaux}

Les Empires coloniaux, répartis ainsi de par le monde, devaient être des sources d'approvisionnement par excellence pour leur métropole, et des marchés privilégiés pour les produits manufacturés métropolitains. Il était donc important d'y avoir une administration efficace et une main-d'œuvre abondante, surtout dans les régions productrices de matières premières et de denrées.

En général, chaque métropole implanta dans ses colonies des structures administratives similaires à celles qu'elle connaissait. On dit souvent que c'était l'équivalent des administrations provinciales métropolitaines. Or, là s'arrête la similitude étant donné la différence d'objectifs à atteindre. Les administrateurs coloniaux, nés pour la plupart en métropole, devaient appliquer des règles rigides édictées à des milliers de kilomètres de là par leurs supérieurs qui ignoraient l'environnement où elles seraient appliquées. En cas d'hésitation ou de refus, ceux-ci perdaient tout simplement leur emploi; mais, en obéissant fidèlement, 